Culture et histoire dans l'espace roman

Crise(s) dans le monde ibérique et ibéro-américain

\title{
La crise argentine en scène : Un momento argentino de Rafael Spregelburd
}

Isabelle Clerc

\section{(2) OpenEdition}

1 Journals

Édition électronique

URL : https://journals.openedition.org/cher/3922

DOI : 10.4000/cher.3922

ISSN : 2803-5992

Éditeur

Presses universitaires de Strasbourg

\section{Édition imprimée}

Date de publication : 1 décembre 2015

Pagination : 231-240

ISBN : 978-2-86820-913-9

ISSN : 1968-035X

\section{Référence électronique}

Isabelle Clerc, "La crise argentine en scène : Un momento argentino de Rafael Spregelburd», reCHERches [En ligne], 15 | 2015, mis en ligne le 01 décembre 2021, consulté le 22 décembre 2021. URL : http://journals.openedition.org/cher/3922 ; DOI : https://doi.org/10.4000/cher.3922

\section{(c) (i) (2)}

Ce(tte) œuvre est mise à disposition selon les termes de la Licence Creative Commons Attribution Pas d'Utilisation Commerciale - Partage dans les Mêmes Conditions 4.0 International. 


\title{
La crise argentine en scène: Un momento argentino de Rafael Spregelburd
}

\author{
ISABELLE CLERC \\ Université Nice Sophia Antipolis
}

\begin{abstract}
Agentine, décembre 2001.
Ale monde entier découvre avec stupeur un pays jadis parmi les plus grands producteurs et exportateurs de viande et de céréales en proie à la misère, la malnutrition, la faim. L'Argentine est en faillite. Les scènes de pillage, de violence, de chaos se multiplient. Des manifestations ont lieu dans tout le pays, d'une ampleur inédite. La population explose. Létat de siège est décrété. La mobilisation populaire s'accentue, défiant le pouvoir et les forces de l'ordre. La répression s'intensifie. À la crise sociale, économique et financière, fruit de deux décennies de politiques néolibérales et d’endettement croissant, s'ensuit une crise politique: la pression de la rue fait tomber le président De la Rúa, élu démocratiquement deux ans auparavant; les présidents se succèdent jusquà la nomination du sénateur Eduardo Duhalde qui restera une année au pouvoir.

C'est dans ce contexte de crise généralisée que Rafael Spregelburd ${ }^{1}$ a entrepris l'écriture de Un momento argentino. Il a construit la pièce autour de deux situations qu'il avouera plus tard avoir choisies de façon très arbitraire: quatre amis (deux militaires et leur femme) se retrouvent pour regarder les diapositives d'un voyage à Cuba réalisé par l'un des deux couples. À cette situation initiale vient interférer une seconde à travers Alicia, fille de l'autre couple, qui avoue à ses parents prendre part aux cacerolazos qui ont lieu dans le pays et s'associer

1 Dramaturge argentin né en 1970 à Buenos Aires. Parmi ses principales pièces on peut citer La tiniebla (1994), Remanente de invierno (1995), Heptalogía de Hieronymus Bosch commencée en 1997, Fractal. Una especulación científica (2000), Bizarra. Una saga argentina, pièce fleuve en dix épisodes écrite en 2003, Lúcido (2006), etc. Son écriture est marquée par une activité qui touche tous les domaines du théâtre: il est auteur, acteur, metteur en scène (il réalise toujours la mise en scène de ses textes), traducteur, et a écrit de nombreux articles théoriques sur le théâtre.
\end{abstract}


à leurs revendications. Elle devient même leur porte-parole en se transformant en bombe humaine afin d'obtenir l'exécution de leurs revendications. Un des personnages essaie de désamorcer la bombe mais en déconnectant un fil il accélère le compte-à-rebours. La pièce se termine alors que l'explosion de la bombe est imminente.

Cette pièce écrite à chaud sur la situation que traverse l'Argentine fait figure d'exception dans la trajectoire du jeune dramaturge argentin qui avait pris ses distances vis-à-vis de ce théâtre en prise avec l'actualité ou qui prétend faire passer un message; une norme dans les années 1970-1980. «El mejor teatro es el que se refiere a la realidad en general y no a la actualidad periodística en particular» dit-il en $1995^{2}$.

Considera que su generación ha logrado recuperar una situación gozosa del teatro, liberado del imperativo de «decir lo importante» de los 80 , un teatro que propone un encuentro festivo, pero de una enorme responsabilidad con el presente. «El teatro es una forma de conocimiento de lo real, pero una forma lúdica, no periodística», afirma, y defiende el derecho de los argentinos a la ficción, ante la demanda de teatro ideológico que a veces recibe del exterior. (Rodríguez Ballester 2007).

C’est précisément pour répondre à une demande de ce type qu'est né Un momento argentino: face à la grave crise que traversa l'Argentine à la fin de l'année 2001, le Royal Court Theatre de Londres demanda à Rafael Spregelburd d'écrire une pièce brève en vue d'intégrer sa programmation internationale de lectures sur la défense des droits de l'homme. C'est avec beaucoup de scepticisme qu'il accepta les doubles contraintes décriture qui lui étaient imposées: rendre compte de ce que le pays était en train de vivre tout en abordant le thème des droits de l'homme. Les violences commises par les forces de l'ordre contre les manifestants, et qui firent une trentaine de victimes et des dizaines de blessés (sans compter les centaines d'arrestations), montrent à elles seules que les deux thématiques sont indissociables.

Le texte de la pièce fut accompagné d'un long prologue écrit à destination du théâtre londonien et dans lequel l'auteur apporte quelques éclaircissements sur les conditions d'écriture. L'édition postérieure du texte de la pièce inclut ce prologue ainsi qu'une note finale dans laquelle Rafael Spregelburd évoque le processus de création, à la fois du texte et de la mise en scène, puisque la pièce a ensuite été représentée dans trois villes européennes ${ }^{3}$. D’autres textes et annotations seront joints, formant un paratexte dense et détaillé dans lequel le récepteur peut trouver des clés de lecture ainsi que des orientations sur la façon dont la pièce doit être perçue.

2 Cité par Dubatti 2006: 175.

3 À la suite de la lecture au Royal Court Theatre la pièce a été représentée au Old Vic Theatre de Londres en août 2002, à Stockholm en novembre 2002, puis à Stuttgart en décembre 2002, dans des mises en scène de Rafael Spregelburd, différentes selon les villes puisque la pièce a toujours été jouée dans la langue des pays d'accueil. 
Dans le prologue, Rafael Spregelburd fait part de ses difficultés à écrire sur des événements si récents: «Estamos demasiado cerca de lo que pretendemos describir, aunque sea con la lente deformante del teatro» (Spregelburd 2003: 136). Ces difficultés sont en grande partie liées à sa conception du théâtre:

Un teatro independiente, un fenómeno autónomo, y contracultural, que desprecia el sentido común, que se aleja de la tarea didáctica de transmitir mensajes, como si los artistas fueran iluminados conocedores de la verdad y tuvieran la misión de bajar esta verdad a un pueblo iletrado e ignorante. (Spregelburd 2003: 143)

À la lecture du prologue, on devine que l'écriture de cette pièce a été un véritable défi pour Rafael Spregelburd. Un momento argentino permet donc de s'interroger sur les moyens dont dispose l'écriture théâtrale pour parler de la réalité, immédiate ou non. C'est la question qu'il pose dans le prologue:

¿Qué relación puede haber entre el teatro y la defensa de los derechos humanos? ¿Qué relación puede haber entre un "tema", un tema de la realidad, y los procedimientos de fabricación de ficción teatral?» (Spregelburd 2003: 142).

Dans son ouvrage Les théâtres $d u$ réel. Pratiques de la représentation dans le théâtre contemporain, Maryvonne Saison analyse les difficultés de la scène contemporaine à représenter le réel, tâche pourtant inéluctable car inhérente à cet art de la représentation qu'est le théâtre ${ }^{4}$ :

Quelles que soient les esthétiques, l'enjeu d'une recherche artistique est précisément, avant toute considération particulière, de définir la modalité de sa référence à la contemporanéité et de caractériser le réel qu'elle se donnera pour tâche (même impossible) de représenter. (Saison 1998: 13)

À quelle réalité se réfère Un momento argentino? Quelle représentation de la crise, ou plus largement du réel, propose la pièce? Selon quelles modalités? Pourquoi l'entreprise semble-t-elle si ardue?

Fuyant tout réalisme, Rafael Spregelburd a choisi le mode de la farce, « recurso [...] en el que los actores enuncian que son actores" (Spregelburd 2003: 149): «En principio, no he encontrado otra forma posible que no fuera la farsa. La farsa reproduce mejor que cualquier otro género mi desazón y mi sombría sorpresa ante los hechos que vive mi país» (Spregelburd 2003: 136). C'est par ce procédé de distanciation que débute la pièce: un homme apparaît, se présente (sous un nom différent de celui indiqué dans le texte, les didascalies précisant que le nom importe peu et qu'il peut même être celui de l'acteur), explique au public qu'il est un acteur et que dans quelques instants il va entrer dans la peau d'un personnage. Les quatre autres acteurs entrent en scène et se présentent à leur tour, observent le public, se préparent et s'installent avant le début de la représentation. Ángel enfile son costume de militaire sous les yeux du public, Guillermo explique quel personnage il va interpréter, Cuca parle de sa nervosité, semblable à celle que ressent tout acteur avant d'entrer en scène, Guillermo indique à l'actrice qui joue

4 Représentation ici dans le sens de représenter, rendre présente à l'esprit une réalité donnée. 
le rôle de sa fille la posture qu'elle doit adopter, etc. Les acteurs-personnages annoncent que nous sommes au théâtre; on peut d'ailleurs se demander qui, du personnage ou de l'acteur, parle au début de la pièce, si nous sommes déjà dans la fiction ou dans le réel. On ne sait pas véritablement, en fait, quand commence la fiction, quand débute la représentation. Ces acteurs qui entrent en scène en faisant croire au public que la pièce va bientôt commencer, que dans quelques instants ils vont jouer la comédie, alors qu'ils sont déjà en train de le faire, tout cela ne peut qu'entrer en résonance avec une autre comédie, celle du pouvoir et de la politique en Argentine, que la population décida de dénoncer et de rejeter en bloc à la fin de l'année 2001. Il n'y a pas de frontière entre l'acteur et son personnage, entre l'acteur et le militaire ici, comme il n'y en a pas entre l'homme politique et l'acteur. La politique argentine est une farce et la classe politique est faite d'acteurs qui jouent le rôle de représentants politiques honnêtes. Le procédé de distanciation, les oscillations entre fiction et réalité, le double niveau de représentation que la pièce installe dès le début rappellent combien la théâtralité a envahi nos sociétés. La vie quotidienne, et plus particulièrement la vie politique, est une constante mise en scène ${ }^{5}$.

Revenons un instant sur la crise argentine de 2001 et notamment sur les raisons de cette incroyable explosion populaire. Car le fait marquant de cette crise, plus que l'effondrement de son économie, est l'ampleur de la protestation citoyenne - en particulier de la classe moyenne - et ce quelle nous dit. Un des éléments qui expliquent l'explosion populaire de cette fin d'année 2001, en plus des mesures comme le corralito et la fin de la convertibilité (parité peso-dollar), est l'exaspération de la population, un ras-le-bol général face aux révélations quotidiennes de vol, de mensonge, de corruption de la classe politique. Dans son analyse de la crise, Victor Armony avance l'idée que le premier coupable du désastre argentin, avant les "élites économiques» qui "ont prêché le libreéchange tout en saignant l'État», ont "appuyé toutes les dictatures militaires et en ont profité sans aucune gêne pour s'enrichir», et avant les «organismes financiers internationaux, particulièrement le FMI», est la «classe politique» (Armony 2004: 13). Létendue de la contestation populaire exprime indéniablement un rejet de la classe politique, une crise de la représentation politique:

La coupure entre la politique et la citoyenneté correspond à une panne de la représentation dans le double sens du terme: la représentation-mandat (le principe de médiation entre l'État et les citoyens) et la représentation-figuration (la production d'une image de la totalité sociale, dans laquelle les citoyens se reconnaissent). Les Argentins ne se sentent plus représentés par leurs leaders, ils considèrent que le pacte entre le peuple et ses dirigeants a été rompu. [...] Beaucoup d'Argentins de classe moyenne se sentent trahis par les politiciens, mais [...] leur colère émane aussi d'un malaise plus profond: c'est une certaine image

5 Référence à l'ouvrage du sociologue Erving Goffman. Les événements récents en Argentine, les mensonges répétés de la classe politique illustrent parfaitement cette «mise en scène de la vie quotidienne». 
d'eux-mêmes et de l'Argentine qui a été radicalement mise en cause. (Armony 2004: 151-152).

Armony relève un aspect clé de cette crise de la représentation: le mythe d'une Argentine promise à un avenir brillant, profondément ancré dans l'imaginaire argentin, s'est effondré avec la crise. On perçoit mieux le défi qu'a constitué pour Rafael Spregelburd l'écriture de la pièce: comment donner une représentation de l'Argentine en crise alors que c'est la représentation même qui fait défaut? $\mathrm{La}$ "panne de la représentation» dont parle Victor Armony est précisément au cœur de Un momento argentino. Plusieurs éléments renvoient à la méfiance de la population vis-à-vis de la classe politique. Au début de la pièce, l'acteur qui incarne le personnage d'Ángel justifie ses interventions en ces mots: «acá nadie pretende engañar a nadie»(Spregelburd 2003: 153). Et Guillermo d'ajouter, presque simultanément: «no, no, no vamos a engañar a nadie» (Spregelburd 2003: 153). Ces phrases peuvent en rappeler d'autres et entrent en résonance avec les discours politiques, les promesses non tenues... On se souviendra du célèbre «no los voy a defraudar» de Carlos Menem qui a rythmé sa campagne électorale pour l'accession à la présidence de la République argentine en 1989 et qui, au vu de la politique qu'il a menée au cours de ses deux mandats, apparaît comme un bel exemple de mensonge politique. Le cynisme de l'ancien président de la République ne s'est d'ailleurs pas arrêté là puisqu'il s'est représenté aux élections présidentielles de 2003 avec le même slogan. Le procédé de distanciation renvoie inévitablement à la comédie du pouvoir en Argentine et aux sentiments de la population.

Quant à Alicia, elle incarne le peuple et, en particulier, cette classe moyenne qui manifeste massivement fin 2001 et ne croit plus en ces hommes politiques qui les gouvernent. Comme de nombreux Argentins à l'époque, c'est la première fois quelle participe à une manifestation. Son engagement aux côtés de la population révèle une coupure entre politique et citoyenneté: «iQué me importa a mí la política! ¡Esto no es política! ¡Esto es la gente, es la vida de la gente! ¡La vida se agota!» (Spregelburd 2003: 168). La «política» et la «gente» seraient devenus indissociables. C'est ce que montre Armony dans son analyse de la crise argentine. La «gente», c'est l'acteur de la contestation, nouvel acteur de la politique qui peut avoir un poids décisif comme l'ont montré les événements de décembre 2001; des gens, des citoyens dont «la participation à la vie publique [a un] caractère spontané [et qui] ne répondent pas à une idéologie, à un parti ou à un leadership donné. Ils sont "des gens ordinaires"» (Armony 2004: 166). Les citoyens ne se reconnaissent plus dans la politique et ses représentants, raison pour laquelle s'est accentuée avec la crise une autre forme de participation à la vie politique, déconnectée du système des partis. Les assemblées populaires et autres formes de démocratie participative se sont en effet considérablement développées.

Si crise de la représentation il y a, si l'ancrage dans le réel et dans l'actualité est clair et explicite, il n'est pas développé davantage. La pièce ne parle que par bribes 
de la crise argentine. Il y a bien ces phrases distillées çà et là et prononcées par Alicia sur les cacerolazos, sur les revendications des manifestants, sur ce qu'il se passe dehors; il y a bien quelques références précises au contexte qui établissent une connexion avec la réalité immédiate mais les autres personnages font tout pour ne pas les entendre, pour éluder une réalité qui visiblement les dérange. Un des personnages va jusqu'à interpeller l'actrice qui joue le rôle d'Alicia par son vrai nom afin qu'elle se taise.

Dans la pièce la fable passe au second plan. Il n'y a pas de fiction à proprement parler: au début rien ne se passe, les dialogues fonctionnent sur du vide; les diapositives, qui pourraient contenir des messages, raconter ce que le texte ne dit pas, éclairer, informer le spectateur, ne renvoient à rien de concret. Et les commentaires des personnages sur ce qu'ils voient sont incompréhensibles et souvent sans lien avec l'image. D'ailleurs, dans une longue didascalie il est dit que le contenu de ces diapositives importe peu, quelles peuvent tout aussi bien représenter une Ford Falcon qu'un drapeau argentin ou un animal, ce qui montre bien que les personnages sont déconnectés de la réalité, puisqu'ils peuvent observer et commenter indifféremment un des symboles de la répression durant la dictature (la Falcon) et un animal quelconque. La seule véritable histoire que raconte la pièce est celle d'Alicia, enfant volée durant la dictature, dont les parents ont été torturés et tués, et qui participe aux cacerolazos. Mais son histoire et ses revendications sont mises à mal par les autres personnages comme nous allons le voir.

Il semble y avoir une difficulté à représenter le réel. À l'évidence le récepteur ne trouvera dans la pièce aucune vérité immuable. Il y est bien dit un certain nombre de choses de la réalité mais aucune lecture interprétative du réel n'est proposée. Nous en avons une première illustration avec la «chica-bomba» qui prend part aux cacerolazos, et menace de se faire exploser si les revendications des manifestants, dont voici un extrait, ne sont pas exécutées sur-le-champ:

ALICIA. - [...] todos quietos, tienen cinco minutos para: (Saca una lista de un bolsillo) Uno: que renuncie la Corte Suprema de Justicia, Dos : que nos dejen sacar la plata de los bancos, que es nuestra, Tres: que investiguen al jefe de la policía que dio la orden de reprimir la semana pasada... y la que viene, Cuatro: creen nuevos puestos de trabajo [...] Ocho: asamblea popular y soberana, ¡ya! Nueve: no al pago de la deuda externa, condenar ya a los usureros. Diez: repatriación de los fondos de los bancos nacionales que huyeron al exterior en diciembre. Once: expropiación de los capitales de los bancos y usureros privados para devolverle a la gente sus ahorros de toda una vida. Doce: juicio a los militares que vendieron armas ilegales durante la guerra de Perú y Ecuador, incluido el ex presidente de la Nación. Trece: restitución de todos los niños robados durante la dictadura a sus familias legítimas. Catorce: encarcelamiento de todos los militares cuya participación en estos hechos ya ha sido sobradamente demostrada... (Spregelburd 2003: 166)

L'ampleur des revendications, légitimes pour la plupart et similaires à celles entendues lors des manifestations de décembre 2001, et l'impossibilité de les appliquer dans le laps de temps indiqué les rendent ridicules. La maladresse des 
manifestants renvoie aussi au caractère spontané et inédit de cette contestation populaire, déconnectée des partis politiques traditionnels et de toute démarche militante, que l'auteur tourne ici en dérision pour offrir au lecteur/spectateur une scène où l'humour, ressort de la théâtralité, est un ingrédient supplémentaire de la farce et ôte toute crédibilité aux manifestants.

Labsence de jugement de la part de l'auteur est patente dans cet autre exemple que l'on trouve à la fin de la pièce: alors que la tension est grande - en tentant de désamorcer la bombe Ángel a créé un court-circuit qui a accéléré le compte à rebours - ce dernier raconte à Alicia qu'il a lui-même torturé et tué ses parents biologiques. Le lecteur/spectateur apprend presque incidemment qu'Alicia est une enfant de disparus et qu'elle a été adoptée par le militaire Guillermo et sa femme grâce au colonel Ángel. La question des droits de l'homme en Argentine - à laquelle la pièce devait référer, rappelons-le - s'illustre par la torture durant la dictature et le sort des disparus. La présence de cette thématique n'est pas fortuite. Rafael Spregelburd explique bien dans son prologue que de nos jours en Argentine on torture dans les commissariats avec la picana; la question des disparus et des bébés volés durant la dictature est, elle aussi, toujours d'actualité. Mais c'est l'association avec la crise de 2001 qui est intéressante. La dictature du «Processus de réorganisation nationale» (1976-1983) et la crise de 2001 sont bien liées car c'est dans la première que l'on trouve les germes de la crise: le système financier qui fera faillite, le début de l'endettement, la mise en place de politiques néolibérales sont à mettre sur le compte des militaires. D’où, certainement, leur présence sur scène, qui rappelle peut-être aussi que la menace est grande d'un retour des forces armées aux commandes du pays ${ }^{6}$.

La terrible révélation d’Ángel sur un crime commis durant la dictature, ce "gran momento argentino» comme dit le texte, passe presque au second plan du fait de l'explosion de la bombe que le rythme du compte à rebours rend imminente et pesante. Cet instant de vérité, recherchée par Alicia comme par toutes les familles de disparus, est ici privé de la solennité que la gravité du sujet pourrait requérir puisque, immédiatement après ses aveux, Ángel fait part à Alicia de son envie pressante d'uriner. Ne pouvant lâcher les câbles par peur de faire exploser la bombe (il fait dorénavant office de masse) il demande à Alicia de l'aider à uriner dans un pot de fleurs. Précisons que, sous la menace de l'explosion, les trois autres personnages ont lâchement quitté les lieux, laissant Alicia avec l'ancien tortionnaire de ses parents. Le "gran momento argentino" tant attendu n'a pas lieu. L'extrême trivialité des besoins physiologiques d’Ángel, expression du bas matériel et corporel de Bakhtine, comme le recours à l'humour ou à l'exagération, balaie tout message critique de dénonciation. D’ailleurs Ángel parvient facilement à faire reculer Alicia sur son engagement, même s'il est trop tard. En aidant Ángel à uriner dans le pot de fleurs comme il le lui a demandé, Alicia montre sa faiblesse de caractère, son manque de pugnacité, d’engagement.

6 Au moment des émeutes, le président De la Rúa avait fait appel à l'armée pour rétablir l'ordre dans le pays; celle-ci avait refusé d'intervenir. 
Elle ne va pas au bout de ses convictions. Ce personnage, incarnation des enfants volés de la dictature, n’est à aucun moment idéalisé ni présenté comme une victime. De la même façon, le personnage du militaire n'a rien de l'archétype du tortionnaire, hormis son caractère autoritaire: du fait de ses interventions métathéâtrales répétées, une complicité s'est instaurée avec le lecteur/spectateur qui peut même par moments le trouver sympathique ${ }^{7}$. La pièce nénonce pas de vérités connues a priori - "verdades conocidas a priori» - (Spregelburd 2003: 143). C’est ainsi que Rafael Spregelburd conçoit le théâtre: "El teatro que no afirma, sino que pregunta. [...] El teatro que nos enfrenta a las cosas del mundo sin orientar su significación» (Spregelburd 2003: 143). C’est au spectateur de se faire sa propre idée.

Faut-il voir dans tout cela une impossibilité à dire le monde? N'est-ce pas ce que révèle le recours au procédé de distanciation? L’idée de cette esthétique théâtrale est de casser l'illusion, de dévoiler au spectateur les mécanismes de la représentation, de montrer que nous sommes au théâtre, que tout n'est que fiction; c'est un théâtre qui parle de théâtre et ne cherche pas à se faire passer pour le réel:

la forme épique [...] mettra au point des procédures qui permettent de casser l'illusion théâtrale, de ramener le spectateur à la conscience de soi et au sentiment que ce qui lui est donné à voir n’est pas la réalité, ni même une copie parfaite, mais une «représentation», une image insolite, problématique et dépourvue de «naturel» puisqu'elle avoue sa nature théâtrale... (Roubine1998: 139)

Le spectateur est face à une représentation, ou ce que lon veut nous faire passer comme tel. Représentation à laquelle il ne croit plus ou qui n'est plus. Lidée de crise de la représentation est sous-jacente. Maryvonne Saison pose le problème au début de son ouvrage:

La crise du théâtre (sans doute pourrait-on dire de l’art en général) est intimement liée à la crise du politique, mais celle-ci renvoie à son tour à un bouleversement global des modes de penser: c'est la représentation elle-même qui est devenue aléatoire; l'idée s'impose qu'il faut faire son deuil de toute représentation d'une réalité devenue éclatée et chaotique. (Saison 1998: 38)

C'est un constat partagé par Rafael Sregelburd dans son analyse du théâtre porteño:

Es un teatro paradójico que se basa, a mi entender, en la crisis de la representación. La crisis de la representación como medio de conocimiento y de verdad. Esta crisis es, a mi entender, fruto del fracaso de nuestras democracias berretas y corruptas. La democracia supone que el pueblo gobierna para sí mismo, a través de sus representantes. Pues el pacto representativo se ha roto. Toda representación entraña -a los ojos de los argentinos- una tácita vinculación con el Mal. No tenemos confianza en la representación y sus mecanismos, y demandamos cada

7 On retrouve ici une thématique développée entre autres par Eduardo Pavlovsky dans quelques-unes de ses pièces: malgré ses actes d'une monstruosité extrême, le tortionnaire est un homme comme les autres. Nous renvoyons le lecteur à notre travail sur la banalité du mal dans les œuvres de ce dramaturge argentin (Clerc 2012). 
vez más ver la cosa en sí misma, la presentación de la cosa, y no su mediatización vergonzosamente deformante, estilizada o simbólica. (Spregelburd 2003: 142)

Spregelburd ne fait pas pour autant le deuil de la représentation: pour lui le théâtre le plus intéressant à ses yeux "asume los riesgos de la representación, delatando que el objeto representado bien podría estar vacío» (Spregelburd 2003: 143).

Qu'il soit vide ou incomplet, l'objet est en tout cas fragmenté. Sur le mode ludique, Rafael Spregelburd opère une déconstruction de tous les procédés dramaturgiques fondés sur l'illusion. Il crée un monde scénique en décomposition où les acteurs ne peuvent tenir, confinés dans leur rôle, et où même le décor s'effondre peu à peu ${ }^{8}$; à l'image de cette Argentine du début du $\mathrm{XXI}^{\mathrm{e}}$ siècle. Le lecteur/spectateur se trouve face à un univers dramatique singulier où le personnage est acteur, où la réalité se joue de la fiction, et vice-versa. Par la distanciation, l'interaction entre fiction et réalité, le dramaturge interpelle le public, l'invite à participer à l'univers fictionnel de la pièce. Il démonte les mécanismes de la représentation pour finalement laisser au lecteur/spectateur le soin de les «remonter» selon sa propre interprétation. C'est à lui qu'il revient de construire un sens.

Si la pièce s'amuse à montrer les mécanismes de la représentation, et donc que le monde créé n'est que mensonge, elle s'achève pourtant sur un acte qui ne peut se cacher derrière aucun artifice, un acte d'une vérité irréfutable, peut-être le seul de la pièce: l'acteur qui incarne Ángel et qui, de façon répétée, sort de son rôle pour rappeler que l'on est au théâtre, urine en effet véritablement sur scène. Cette action finale, d'une extrême trivialité, voit se fondre fiction et réalité et ébranle du même coup tout ce que la pièce a mis en place jusqu'à cet instant. L'urine déversée sur scène condense tout à la fois la représentation de la crise et la crise de la représentation, dans toutes ces acceptions.

\section{Bibliographie}

Armony, V., 2004, L'énigme argentine. Images d'une société en crise, Outremont Québec, Athéna éditions.

Clerc, I., 2012, "La banalité du mal” selon Eduardo Pavlovsky», Babel, 26, p. 299-312.

Dubatti, J., 2006, «Hacia una relectura post-postmoderna del teatro argentino: notas sobre Rafael Spregelburd», Revista Nuestra América, 2, p. 171-181.

Figueroa, J., 2003, «El orgullo perdido en Argentina», (http://www.tmg-online. org/download/figueroa_momento_argentino.doc).

8 Dans la mise en scène réalisée en Allemagne, un acteur devait chaque soir casser un élément du décor (Figueroa 2003). 
Goffman, E., 1973, La mise en scène de la vie quotidienne. 1. La présentation de soi. 2. Les relations en public, Paris, Éditions de Minuit.

Rodríguez Ballester A., 2007, "Cómo curarse de la realidad con teatro », Clarín, Revista N., 14/4/2007 (version en ligne: http://edant.clarin.com/suplementos/ cultura/2007/04/14/u-03411.htm).

Roubine J.-J., 1998, Introduction aux grandes théories du théâtre, Paris, Dunod.

Saison M., 1998, Les Théâtres du réel. Pratiques de la représentation dans le théâtre contemporain, Paris, L'Harmattan.

Spregelburd R., 2003, «Un momento argentino», in: Jorge Dubatti (comp.), Nuevo teatro argentino, Buenos Aires, Interzona, p. 133-179.

Spregelburd R., 2003, «Prólogo» et «Nota final/ Antes y después de esta obra», in: "Un momento argentino", in: Jorge Dubatti (comp.), Nuevo teatro argentino, Buenos Aires, Interzona, p. 136-148 et p. 173-179. 